\title{
A Simple Option Formula For GENERAL JUMP-DIFFUSION AND OTHER EXPONENTIAL LÉVY PROCESSES
}

\author{
ALAN L. LEWIS* \\ Envision Financial Systems \\ and OptionCity.net
}

August, 2001

Revised: September, 2001

\begin{abstract}
Option values are well-known to be the integral of a discounted transition density times a payoff function; this is just martingale pricing. It's usually done in ' $S$-space', where $S$ is the terminal security price. But, for Lévy processes the $S$-space transition densities are often very complicated, involving many special functions and infinite summations. Instead, we show that it's much easier to compute the option value as an integral in Fourier space - and interpret this as a Parseval identity. The formula is especially simple because (i) it's a single integration for any payoff and (ii) the integrand is typically a compact expressions with just elementary functions. Our approach clarifies and generalizes previous work using characteristic functions and Fourier inversions. For example, we show how the residue calculus leads to several variation formulas, such as a well-known, but less numerically efficient, 'Black-Scholes style' formula for call options. The result applies to any European-style, simple or exotic option (without pathdependence) under any Lévy process with a known characteristic function.
\end{abstract}

\footnotetext{
* All comments welcome and encouraged.

Contact info. : Tel: (949) 720-9614, email: alanlewis@home.com

983 Bayside Cove West, Newport Beach, Ca 92660 USA
} 


\section{INTRODUCTION AND SUMMARY}

The benchmark model for security prices is geometric Brownian motion. A relatively simple yet powerful generalization is the class of all continuous-time process where all non-overlapping increments $X_{t_{2}}-X_{t_{1}}=\log \left(S_{t_{2}} / S_{t_{1}}\right)$ are independent random variables with stationary distributions. This set of processes $X_{t}$ are the Lévy processes or processes with stationary independent increments. [see the monographs by Sato (1996) and Bertoin (1999)]. They consist of a combination of a linear drift, a Brownian motion, and an independent jump process Here we provide a solution to the associated European-style option valuation problem.

These models help explain some, but not all, of the well-documented deviations from the benchmark model. Lévy process models can be good fits to daily stock return distributions which are characterized by wide tails and excess kurtosis. [For example, see Eberlein, Keller, and Prause (1998)]. The so-called jump-diffusions (which are members of the class where the jumps are compound Poisson processes) offer a compelling explanation for the relatively steep smiles observed in expiring index options like the S\&P500. ${ }^{1}$ It should be noted that the independent increment assumption is counter-factual in some respects. ${ }^{2}$ Nevertheless, because of their successes, flexibility, and analytic tractability, continued financial applications are likely. For a recent survey of applications, in finance and elsewhere, see Barndorff-Nielsen, Mikosch, and Resnick (2001).

Jump-diffusion models are distinguished by their jump amplitude distributions. Two examples are Merton (1976) who solved for option prices with log-normally distributed jumps, and Kou (2000), who did the same for a double exponential distribution. To obtain an option formula, the authors relied upon particular properties of those distributions. Merton's solution relies upon a product of lognormal variates being lognormally distributed. Kou's derivation stresses the importance of the memoryless property of the exponential distribution. Our results make clear that, in fact, no special properties are needed: we obtain an option formula for any jump distribution.

In addition, many of the original results for these models are very complicated. Special functions and complicated expressions are required when option formulas are given in ' $S$-space' or stock price space. ${ }^{3}$ However, more recently, it has been recognized that option values for both the Lévy process problem and related proportional returns problems are much simpler in Fourier space ${ }^{4}$. For example, Carr and Madan (1999) have derived relatively simple formulas for call options on Lévy processes, working in Fourier space. Bakshi and Madan (2000), although not working directly on Lévy processes, derive an applicable 'Black-Scholes-style' formula for call options using characteristic functions and some more complicated formulas for general claims. Raible 
(2000) obtained an option formula which is very similar to our general result (see below). However, he presents it as a mixture of Fourier and two-sided Laplace transforms. Because we use the generalized Fourier transform consistently, our strip condition is more transparent. In Lewis (2000), we obtained related inversion formulas for options under stochastic volatility, a proportional returns problem. Here we generate the value of the general claim under a Lévy processes as an integral of Fourier transforms. Once you have our main result, the residue calculus provides a standard approach to variations. For example, we show that the BlackScholes style formula is simply obtained by moving integration contours.

The formula can be easily explained with a little background. Assume that under a pricing measure a stock price evolves as $S_{T}=S_{0} \exp \left[(r-q) T+X_{T}\right]$, where $r-q$ is the cost of carry, $T$ is the expiration time for some option, and $X_{T}$ is some Lévy process satisfying $\mathbb{E}\left[\exp \left(X_{T}\right)\right]=1$. For Lévy processes, the important role of the characteristic functions $\phi_{t}(u)=\mathbb{E}\left[\exp \left(i u X_{t}\right)\right], u \in \mathbb{R}$ is well-known. Like all characteristic functions, they are Fourier transforms of a density and typically have an analytic extension (a generalized Fourier transform) $u \rightarrow z \in \mathbb{C}$, regular in some strip $\boldsymbol{S}_{X}$ parallel to the real $z$-axis.

Somewhat less appreciated, yet key to our approach, is the recognition that option payoff functions also have simple generalized Fourier transforms. Using the variable $x=\log S_{T}$, these transforms are $\hat{w}(z)=\int_{-\infty}^{\infty} \exp (i z x) w(x) d x$, where $w(x)$ is the payoff function ${ }^{5}$. For example, if $K$ is a strike price, the call option payoff is $w(x)=\left(e^{x}-K\right)^{+}$and so, by a simple integration, $\hat{w}(z)=-K^{i z+1} /\left(z^{2}-i z\right), \operatorname{Im} z>1$. Note that if $z$ were real, this regular Fourier transforms would not exist. As shown in Lewis (2000), payoff transforms $\hat{w}(z)$ for typical claims exist and are regular in their own strips $S_{w}$ in the complex $z$-plane, just like characteristic functions. Then, the initial option value $V\left(S_{0}\right)$ is given by simply integrating the (conjugate) product of these two transforms times a phase factor. To do the integration legally, one has to keep $z$ within the intersection of the two strips of regularity $\boldsymbol{S}_{w}$ and $\boldsymbol{S}_{X}^{*}\left(\boldsymbol{S}_{X}^{*}\right.$ is the reflection of $\boldsymbol{S}_{X}$ across the real $z$-axis). With that synopsis, the formula is

$$
\begin{aligned}
& \text { Option values: } \quad V\left(S_{0}\right)=\frac{e^{-r T}}{2 \pi} \int_{i \nu-\infty}^{i \nu+\infty} e^{-i z Y} \phi_{T}(-z) \hat{w}(z) d z, \\
& \text { where } Y=\ln S_{0}+(r-q) T, \quad z=u+i \nu, \quad z \in \boldsymbol{S}_{V}=\boldsymbol{S}_{w} \cap \boldsymbol{S}_{X}^{*} .
\end{aligned}
$$

Although very compact, (1.1) contains (as special cases), the Black-Scholes model, Merton's jump-diffusion model, Kou's jump-diffusion model, and all of the pure jump models that have been introduced. Indeed, it applies to the entire class of exponential Lévy processes which have $\mathbb{E}\left[\exp \left(X_{T}\right)\right]<\infty$. The proof of it and our use of the residue calculus to obtain variations is our primary contribution in this paper. 
Any path-independent European-style payoff, plain vanilla or exotic, may be valued. The expression (1.1) is obviously just a single integral. It's real-valued and readily evaluated ${ }^{6}$. Typically the integrand is a short expression, often with only elementary functions. See Tables 2.1 and 3.1 for examples of $\phi_{T}(z)$ and $\hat{w}(z)$ respectively.

Consider the call option again. Since $\hat{w}(z)=-K^{i z+1} /\left(z^{2}-i z\right)$, the integrand in (1.1) is a regular function of $z$ in the larger strip $S_{X}^{*}$, except for simple poles at $z=0$ and $z=i$. Using the residue calculus, you can move the integration contour around in $S_{X}^{*}$. Of course, you pick up residue contributions if you move contours across (or along!) $\operatorname{Im} z=0,1$. We do this and obtain a number of variations on (1.1) - including the Black-Scholes style formula that we mentioned earlier. Finally, it turns out that $\exp (-i z Y) \phi_{T}(-z)$ is the (conjugate of the) Fourier transform of the transition density for $\log S_{0}$ to reach $\log S_{T}$ after the elapse of $T$. This allows us to interpret (1.1) simply as a Parseval identity. In fact, the proof of that clarifies what space of payoff functions are handled by our theory and which types are excluded.

In the next section, we review some fundamental aspects of Lévy processes, their applications to finance, and their analytic characteristic functions. This material is almost entirely standard and experts in those topics could well skim for our notation and then jump to the proof of (1.1) in Section 3.

\section{BACKGROUND}

\subsection{The Framework}

We consider a marketplace in which a stock price or security price $S_{t} \geq 0$ follows an exponential Lévy process $X_{t}$ (defined below) on a continuous-time probability space $(\Omega, \mathbb{F}, \boldsymbol{Q})$. We stress that $\boldsymbol{Q}$ is a fixed martingale pricing measure. The pricing measure has the same null sets as an 'objective' or 'statistical' measure $\boldsymbol{P}$, and the two measures are related by an unspecified Girsanov change-of-measure transformation ${ }^{7}$. However, $\boldsymbol{P}$ plays no direct role in our discussion - all expectations and stochastic processes are defined relative to $\boldsymbol{Q}$.

A stock buyer receives a continuous dividend yield $q$; she could finance her purchase at the riskless rate of interest $r$. The net financing cost (the cost of carry) is $r-q$. It is convenient to explicitly remove this constant - we then investigate option valuation where $S_{t}=S_{0} \exp \left[(r-q) t+X_{t}\right]$ under $\boldsymbol{Q}$, where $X_{t}$ is a Lévy process. Following Sato (1999), we adopt the following definition: 
Definition (Lévy Process) An adapted real-valued process $X_{t}$, with $X_{0}=0$, is called a Lévy process if:

(i) it has independent increments; that is, for any choice of $n \geq 1$ and $0 \leq t_{0}<t_{1}<\cdots<t_{n}$, the random variables $X_{t_{0}}, X_{t_{1}}-X_{t_{0}}, \cdots, X_{t_{n}}-X_{t_{n-1}}$ are independent;

(ii) it is time-homogeneous; that is, the distribution of $\left\{X_{t+s}-X_{s} ; t \geq 0\right\}$ does not depend upon $s$.

(iii) it is stochastically continuous; that is, for any $\varepsilon>0, \operatorname{Pr}\left\{\left|X_{s+t}-X_{s}\right|>\varepsilon\right\} \rightarrow 0$ as $t \rightarrow 0$.

(iv) as a function of $t$, it is right-continuous with left limits.

Processes that satisfy (i) and (ii) are called processes with stationary (or time-homogeneous) independent increments (PIIS). Some authors (e.g. Bertoin) simply define a Lévy process to be a PIIS process with $X_{0}=0$. Such processes can be thought of as analogs of random walks in continuous time.

To prevent an arbitrage opportunity, the stock price (net of the cost of carry) must be a local martingale under $\boldsymbol{Q}$. In fact, we maintain throughout the stronger assumption: $S_{t}$, net of the carry, is a $\boldsymbol{Q}$-martingale. That is, $\mathbb{E}\left[S_{t}\right]=S_{0} \exp [(r-q) t]$ or $\mathbb{E}\left[\exp X_{t}\right]=1$. For those Lévy processes with $\mathbb{E}\left[\exp X_{t}\right]<\infty$, this normalization can be achieved by a drift adjustment.

Types of Lévy processes. In general, Lévy processes are a combination of a linear drift, a Brownian motion, and a jump process. When jumps occur, $X_{t}$ jumps by $\Delta X_{t}=x \in \mathbb{R} \backslash\{0\}$, (the notation means that we exclude zero as a possible jump amplitude $x$ ). Now consider any closed interval $A \in \mathbb{R}$ that does not contain the origin. Then, the cumulative number of jumps in the time interval $[0, \mathrm{t}]$ with a size that belongs to $A$, call it $N_{t}^{A}$, is a random variable which is also a measure. This integer-valued $\boldsymbol{Q}$-random measure is usually written $N_{t}^{A}=\nu([0, t], A)$. With $A$ fixed, then $N_{t}^{A}$ has a Poisson distribution with a mean value $t \int_{A} \mu(x) d x$. Here we have introduced the Lévy measure $\mu(x) d x$, which measures the relative occurrence of different jump amplitudes [see Sato, 2001, Theorem 1.4].

Two types of Lévy processes with a jump component can be distinguished. In type I (the Poisson case), we have $\int_{\mathbb{R}} \mu(x) d x<\infty$. Then, we can write $\mu(x)=\lambda f(x)$, where $\lambda=\int_{\mathbb{R}} \mu(x) d x$ is the Poisson intensity (the mean jump arrival rate), and $f(x) d x=d F(x)$, where $F(x)$ is a cumulative probability distribution ${ }^{8}$. We will also call this case the jump-diffusion case. An example of this type is Merton's (1976) jump-diffusion model, where $x$ is normally distributed: $\mu(x)=\lambda f(x)=\lambda \exp \left[-(x-\alpha)^{2} / 2 \delta^{2}\right] /\left(2 \pi \delta^{2}\right)^{1 / 2}$. 
In the alternative type II case, $\int_{\mathbb{R}} \mu(x) d x=\infty$ and no overall Poisson intensity can be defined. A simple example is the Lévy $\alpha$-stable process: $\mu(x)=c_{ \pm} /|x|^{1+\alpha}, 0<\alpha<2$, where $c_{ \pm}$are two constants for $x>0$ and $x<0$. Carr and Wu (2000) have proposed a special case of this model for stocks. Notice that in the type II example, the source of the divergence is the failure of $\mu(x)$ to be integrable at the origin: there are too many small jumps. The divergence at the origin is always the source of the integrability failure of $\mu(x)$ in type II models - the Lévy-Khintchine representation (see below) guarantees that $\mu(x)$ is always integrable at large $|x|$.

A general integral representation. One can take a differential of $N_{t}^{A}=\nu([0, t], A)$, writing $d N_{t}=\nu(d t, d x)$ and use these differential random measures in an integration theory [see Jacod and Shiryaev (1987)]. With that theory, one can decompose any Lévy process $X_{t}$ into the form ${ }^{9}$ :

$$
X_{t}=\omega_{h} t+\sigma B_{t}+\int_{0}^{t} \int_{\mathbb{R} \backslash\{0\}}(x \nu(d s, d x)-h(x) \mu(x) d s d x),
$$

where $B_{t}$ is a $\boldsymbol{Q}$-Brownian motion, $\omega_{h}$ and $\sigma$ are constants, and $h(x)$ is a truncation function, to be explained. The Brownian motion and the jump process are independent. This representation is unique in the sense that, once the truncation function is fixed, then there is only one set of characteristics $\left\{\omega_{h}, \sigma, \mu(x)\right\}$ for a given $\left\{X_{t}: t \geq 0\right\}$. If the truncation function is changed, the drift $\omega_{h}$ changes but the pair $\{\sigma, \mu(x)\}$ is invariant. The purpose of the truncation function is to make the integral in (2.1) exist near the origin, where the integrand must be taken as a whole. Such a function is only necessary in some Type II models where $\mu(x)$ diverges as $O\left(1 /|x|^{2+\varepsilon}\right)$, $0<\varepsilon<1$; otherwise it may be set to 0 . When a truncation function is needed, $h(x)$ is required to behave like $x$ near the origin and it is frequently taken to be a bounded function away from the origin. Some popular choices are: (i) $h(x)=x 1_{\{|x|<1\}}$ (Sato); (ii) $h(x)=x /\left(1+x^{2}\right)$ (Lukacs, Breiman). If $\int_{\mathbb{R}}|x| \mu(x) d x<\infty$, then the truncation function need not be bounded and the choice $h(x)=x$ may be convenient. In that special case, we see that (2.1) is the sum of a linear drift, a Brownian motion, and an independent compensated jump-martingale.

\subsection{Analytic Characteristic Functions.}

As an application of (2.1), we show that it leads immediately to the celebrated Lévy-Khintchine representation for the characteristic function $\phi_{t}(z)$. This representation is important to our development because it provides an explicit and simple formula for $\phi_{t}(z)$ for all the Poissontype models and some type II models. First, a definition of $\phi_{t}(z)$ and infinite divisibility, then a remark that we will use later, and then the statement of the theorem. 
Definition (Characteristic Function). For $z \in \mathbb{C}(z$ a complex number $)$ and $a<\operatorname{Im} z<b$, we call $\phi_{t}(z)=\mathbb{E}\left[\exp \left(i z X_{t}\right)\right]$ the characteristic function of the process $X_{t}$.

Remark. Let $p_{t}(x)$ be the transition probability density for a Lévy process to reach $X_{t}=x$ after the elapse of time $t$. For $a<\operatorname{Im} z<b$, the characteristic function of the process is identical to the characteristic function of this transition density, which is also the generalized Fourier transform of the transition density ${ }^{10}$.

$$
\phi_{t}(z)=\mathfrak{F}\left[p_{t}(x)\right] \triangleq \int_{\mathbb{R}} \exp (i z x) p_{t}(x) d x, \quad a<\operatorname{Im} z<b .
$$

Definition (Infinitely Divisible Characteristic Functions) . A characteristic function $\phi_{t}(z)$ is said to be infinitely divisible, if for every positive integer $n$, it is the nth power of some characteristic function.

The characteristic function of Lévy processes are infinitely divisible; this is a simple consequence of the PIIS properties. ${ }^{11}$

THEOREM (Lévy-Khintchine Representation). If $\phi_{T}(z), a<\operatorname{Im} z<b$, is an infinitely divisible characteristic function, then it has the representation

$$
\phi_{T}(z)=\exp \left\{i z \omega_{h} T-\frac{1}{2} z^{2} \sigma^{2} T+T \int_{\mathbb{R} \backslash\{0\}}\left[e^{i z x}-1-i z h(x)\right] \mu(x) d x\right\},
$$

where $\int_{\mathbb{R}} \min \left(1, x^{2}\right) \mu(x) d x<\infty$.

Proof: For a proof (when $z$ is real), we refer to Sato (1999, Theorem 8.1). For an extension to complex $z$, we refer to Lukacs' Theorem below. However, just proceeding formally, it's easy to see how the representation (2.2) follows from the representation (2.1). We will only take the simplest case where the deterministic part of the integral in (2.1) exists on its own. Using that assumption, and the independence of the Brownian motion and the jump process, we have immediately from (2.1):

$$
\begin{aligned}
\mathbb{E}\left[\exp \left(i z X_{T}\right)\right]=\exp & \left\{i z \omega_{h} T-i z T \int_{\mathbb{R} \backslash\{0\}} h(x) \mu(x) d s\right\} \\
& \times \mathbb{E}\left[\exp \left(i z \sigma B_{T}\right)\right] \times \mathbb{E}\left[\exp \left(i z \int_{0}^{T} \int_{\mathbb{R} \backslash\{0\}} x \nu(d t, d x)\right)\right]
\end{aligned}
$$

Now it is well-known that $\mathbb{E}\left[\exp \left(i z \sigma B_{T}\right)\right]=\exp \left(-\frac{1}{2} z^{2} \sigma^{2} T\right)$. It is also well-known ${ }^{12}$ that 


$$
\mathbb{E}\left[\exp \left(i z \int_{0}^{T} \int_{\mathbb{R} \backslash\{0\}} x \nu(d t, d x)\right)\right]=\mathbb{E} \prod_{0<t \leq T} e^{i z x_{t}} 1_{\left\{N_{t} \neq N_{t-}\right\}}=\exp \left(T \int_{\mathbb{R} \backslash\{0\}}\left(e^{i z x}-1\right) \mu(x) d x\right) .
$$

Combining these results yields (2.2).

The Lévy-Khintchine representation has the form $\phi_{T}(z)=\exp [-T \Psi(z)]$, where $\Psi(z)$ is called the characteristic exponent ${ }^{13}$. The normalization $\phi_{T}(z=0)=1$ and the martingale identity $\phi_{T}(z=-i)=1$ imply that $\Psi(0)=\Psi(-i)=0^{14}$. Since, for a sensible stock market model, $\phi_{t}(z)$ must exist at both $z=0$ and $z=-i$, it would be helpful if it existed for all $z$ 'in between'. Indeed, all the examples in Table 2.1 exist for $z$ within a horizontal strip $\boldsymbol{S}_{X}=\{z: a<\operatorname{Im} z<b\}$. Here $a$ and $b$ are real numbers, such that $b>a$. Analyticity in strips is typical, based on this theorem:

THEOREM (Lukacs, 1970, Theorem 7.1.1): If a characteristic function $\phi(z)$ is regular ${ }^{15}$ in the neighborhood of $z=0$, then it is also regular in a horizontal strip and can be represented in this strip by a Fourier integral. This strip is either the whole z-plane, or it has one or two horizontal boundary lines. The purely imaginary points on the boundary of the strip of regularity (if this strip is not the whole plane) are singular points of $\phi(z)$.

Remarks. Because of the representation $\phi_{t}(z)=\exp [-t \Psi(z)]$, singularities of $\phi_{t}(z)$ are singularities of $\Psi(z)$. Hence, an immediate corollary of Lukacs' theorem is that the strips of regularity for the analytic characteristic functions of Lévy processes are time-independent ${ }^{16}$. Clearly, a 'good' Lévy process (good for the purpose of building a stock price model), has an analytic characteristic function regular within a strip: $a \leq \operatorname{Im} z \leq b$, where $a \leq-1, b \geq 0$. If a particular Lévy process is a good one, say at $t=1$, then it is a good one for all $t$. Our option valuation formula only applies to good Lévy processes.

Examples. Example of characteristic functions for Lévy processes that have been proposed for the stock market are shown in Table 2.1. [Three entries are adapted from Raible (2000)]. For each process, there is a constant drift parameter $\omega$, determined by solving $\phi_{t}(-i)=1$. We have already mentioned the first two models in the table, both of which have Brownian motion components and are type I or Poisson types.

The remaining (pure jump) models are all type II. The third table entry is the Variance Gamma process. The option value was obtained by Madan, Carr, and Chang (1998). The process is built up by sampling Brownian motion with drift at random times, time increments which themselves are described by another Lévy process. Clearly, the sampled process is a pure jump process. 
The next table entry is the Normal Inverse Gaussian (NIG) process, another pure jump process applied to stock returns by Barndorff-Nielsen (1998). There are 4 real parameters: $(\alpha, \beta, \omega, \delta)$ where, roughly speaking, $\alpha$ and $\beta$ are shape parameters (steepness, tail decay), and $\omega$ and $\delta$ are drift and scale parameters, respectively. [Also see Lillestøl, (1998)].

Following is the Generalized Hyperbolic process. This pure jump Lévy process incorporates the VG and NIG process as special cases, as well as another special case just called the hyperbolic process. There are 5 real parameters: $(\alpha, \beta, \omega, \delta, \lambda)$ where the first 4 have a similar interpretation as before and $\lambda$ is an additional shape parameter. [For surveys, see Bibby and Sørensen (2001) and the dissertation of Prause (1999)]

The last entry is the (maximally) skewed $\alpha$-stable process of Carr and $\mathrm{Wu}(2000)$. The general $\alpha$-stable Lévy process has four parameters $(\alpha, \beta, \omega, \sigma)$, where $\alpha \in(0,2]$ is the index, $\beta \in[-1,1]$ is a skewness, $\omega \in \mathbb{R}$ is a drift, and $\sigma \geq 0$ is a scale parameter. The transition probability distribution $p_{t}\left(X_{t}=x\right)$ typically has a power-law decay as $x \rightarrow \infty$ when $\alpha<2$ and so $\mathbb{E}\left[\exp \left(X_{t}\right)\right]$ does not exist. However, Carr and $\mathrm{Wu}$ have noted that for $\beta=-1$, which they term maximum skewness, then the decay is more rapid, $\mathbb{E}\left[\exp \left(X_{t}\right)\right]<\infty$, and the characteristic function is given by the entry in the table. Note that (unless $\alpha=1,2$ ) the characteristic function has a branch point singularity at $z=0$ and we need a branch cut. [The authors estimated $\alpha=1.61 \pm .01$, based upon daily option quotes for S\&P500 index option over a year ending in May, 2000. They generally suggest that $\alpha \in(1,2)$ for stock prices]. For a stock price model, we want the characteristic function to be regular for at least $-1<\operatorname{Im} z<0$. Given this, the natural choice is to place the branch cut along the imaginary z-axis extending upward from $z=0$. With that choice, the strip of regularity is $\operatorname{Im} z<0$ as shown in the table.

How are characteristic functions calculated? Many of the Type II models are built up by a subordination of a Brownian motion process and this leads to a determination of their characteristic function from the characteristic function of the driving process. Examples of this type are the Variance Gamma, Normal Inverse Gaussian, and Generalized Hyperbolic models, where the first two are special cases of the last. We refer the reader to the original literature for those calculations. With the Type I models and some Type II models, the Lévy-Khintchine representation provides the answer.

To see that, consider the jump-diffusion models, which are type I models. We need to relate the representation (2.1) to the stochastic differential (SDE) that is the usual starting point for these models. For example, both Merton and Kou's jump-diffusion models may be defined by the SDE 


$$
\frac{d S_{t}}{S_{t^{-}}}=(r-q) d t+\sigma d B_{t}+\int_{\mathbb{R}}\left(e^{x}-1\right)[\nu(d t, d x)-\mu(x) d t d x],
$$

where $\mu(x)$ integrates $\left(e^{x}-1\right)$. Under $(2.3), S_{t}$ (net of the carry) is clearly a local martingale. For every type I model, $\mu(x)=\lambda f(x)$. For example, in Kou's double exponential model, $f(x)=\exp (-|x-\kappa| / \eta) /(2 \eta), 0<\eta<1$, where $\kappa$ and $\eta$ are two real parameters.

Starting from (2.3), it is straightforward ${ }^{17}$ to integrate this general SDE to the exponential form $S_{t}=S_{0} \exp \left[(r-q) t+X_{t}\right]$. The result is again the canonical form (2.1) with $h(x)=0$. Given that result, then the Lévy-Khintchine representation (2.2) provides the following:

$$
\begin{aligned}
& \text { In all Type I models: } \quad \phi_{T}(z)=\exp \left\{i z \omega T-\frac{1}{2} z^{2} \sigma^{2} T+\lambda T(\hat{f}(z)-1)\right\} \text {, } \\
& \text { where } \hat{f}(z)=\mathscr{F}[f(x)], \quad z \in \mathcal{S}_{f} \text {, is the characteristic function of } f(x) .
\end{aligned}
$$

The exponentiation of (2.3) determines the drift. But we can also get it from the martingale identity $\phi_{t}(-i)=1$ applied to (2.4). Both procedures give $\omega=-\sigma^{2} / 2-\lambda[\hat{f}(-i)-1]$. For example, with Kou's model again, $\hat{f}(z)=e^{i z \kappa}\left(1-\eta^{2}\right) /\left(1+z^{2} \eta^{2}\right), \quad-(1 / \eta)<\operatorname{Im} z<(1 / \eta)$. Since $0<\eta<1$, the strip of regularity contains $-1<\operatorname{Im} z \leq 0$ and the model is a good one. This shows how to obtain the second entry in Table 2.1. The first entry is obtained similarly ${ }^{18}$.

We also mentioned that the Lévy-Khintchine representation can also be used effectively in some cases of type II models ${ }^{19}$. An example is the last entry in Table 2.1, which may be calculated from $\mu(x)=c_{ \pm} /|x|^{1+\alpha}$. [See, for example, Breiman (1992, Theorem 9.32)].

\section{THE OPTION FORMULA}

As discussed, characteristic functions $\phi(z)$ are typically regular functions in $z$-plane strips. We next show that the same holds true for the transform of typical option payoff functions.

Using $x=\ln S_{T}$, let $w(x)$ be an option payoff function and let $\hat{w}(z)=\mathscr{F}[w(x)], z \in \mathbb{C}$ be its generalized Fourier transform. For example, consider the call option payoff, $w(x)=\left(e^{x}-K\right)^{+}$, and so by a simple integration

$$
\hat{w}(z)=\left.\left(\frac{\exp [(i z+1) x]}{i z+1}-K \frac{\exp (i z x)}{i z}\right)\right|_{x=\ln K} ^{x=\infty}
$$


The upper limit $x=\infty$ in (3.2) does not exist unless $\operatorname{Im} z>1$. Applying this restriction, then (3.2) is well-defined, in fact, regular in that strip:

$$
\text { Call option: } \quad \hat{w}(z)=-\frac{K^{1+i z}}{z^{2}-i z}, \quad \operatorname{Im} \mathrm{z}>1
$$

This result is typical: option payoffs have Fourier transforms (w.r.t. $\log S_{T}$ ) as long as we admit a complex-valued transform variable. Then, $\hat{w}(z)$ exists for $\operatorname{Im} z$ restricted to an interval; i.e., $z \in \boldsymbol{S}_{w}$, just like a characteristic function. We can go through the same exercise for various standard payoff functions and see what restrictions are necessary for their Fourier transforms to exist. The results are given in Table 3.1 below.

So by introducing generalized transforms, we are able to handle standard payoffs which are unbounded at $x=\infty$ (the call), or constant at $x=-\infty$ (the put) and which have no regular Fourier transform. All of the typical payoff functions that one might encounter in practice are handled. But, not every mathematically possible payoff function has a generalized Fourier transform.

Definition. We say a function $f(x)$ is 'Fourier integrable in a strip' if there exists a pair of real numbers $a$ and $b$, where $-\infty \leq a<b \leq \infty$, such that the generalized Fourier transform $\hat{f}(z)=\mathfrak{F}[f(x)]$ exists and is regular for $z=u+i v, u \in \mathbb{R}$, and $\nu \in(a, b)$.

Our valuation theorem below will apply to payoff functions which are (i) Fourier integrable in a strip, and (ii) bounded for $|x|<\infty \quad(S \neq 0, \infty)$. Let us discuss these two requirements in turn. From the definition above, property (i) means that the payoff functions have no worse than exponential growth in $x$, as $|x| \rightarrow \infty$. That is, there exist an $(a, b)$ such that $w(x) \exp (-\nu x) \rightarrow 0$ as $|x| \rightarrow \infty$ for every $\nu \in(a, b)$. Equivalently, as $x \rightarrow+\infty$, we have $w(x) \exp [-(a+\varepsilon) x] \rightarrow 0$ for every $\varepsilon>0$. Similarly, as $x \rightarrow-\infty$, we have $w(x) \exp [-(b-\varepsilon) x] \rightarrow 0$ for every $\varepsilon>0$. For example, for a call option with strike $K$, the requirement is that $\left(e^{x}-K\right)^{+} e^{-\nu x} \rightarrow 0$, as $x \rightarrow+\infty$. This condition again implies that $\nu \in(1, \infty)$, as we found before. Similarly, for the put option, the requirement is that $\left(K-e^{x}\right)^{+} e^{-\nu x} \rightarrow 0$, as $x \rightarrow-\infty$. This condition implies that $\nu \in(-\infty, 0)$. Note that exponential growth in $|x|$ means power law behavior in $S_{T}$ as $S_{T} \rightarrow 0, \infty$.

Property (ii) arises from a technical requirement (see below) that $w(x) \exp (-\nu x)$ be bounded. We already know this term is bounded at $\pm \infty$ (since it's zero). To keep $w(x) \exp (-\nu x)$ bounded everywhere, we also need $w(x)$ bounded for $|x|<\infty$. This is a very mild restriction, in the sense that it only excludes payoff functions that would be unlikely to be offered in the 
marketplace. For example, consider a payoff that behaves like $\left|x-x_{0}\right|^{-1 / 2}=|\log (S / K)|^{-1 / 2}$ as $S \rightarrow K$, where $0<K<\infty$. While this payoff is integrable with respect to $x$ near $x=x_{0}$, it's not bounded and so excluded. In practice, such a payoff would be a pretty dangerous offering for the seller.

Generalized Fourier transforms are inverted by integrating along a straight line in the complex $z$ plane, parallel to the real axis, with $z$ within the strip of regularity. ${ }^{20}$ For example, fixing $\nu=\operatorname{Im} z$, then the payoff functions are given by:

$$
w(x)=\frac{1}{2 \pi} \int_{i \nu-\infty}^{i \nu+\infty} e^{-i z x} \hat{w}(z) d z, \quad z \in \boldsymbol{S}_{w} .
$$

If $f(x)$ is a complex-valued function of a real variable $x$, then by $f \in L^{1}$, we mean that $\int_{-\infty}^{\infty}|f(x)| d x<\infty$, where $|f|$ is the modulus of $f$. With that notation, we need the following

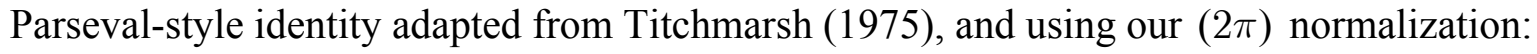

THEOREM 3.1 (Titchmarsh, Theorem 39): Let both $f(x), g(x) \in L^{1}$ and one of them is bounded. Assume that $\int_{-\infty}^{\infty} f^{*}(x-y) g(x) d x$ is continuous at $y=0$. In addition, assume that, with $u \in \mathbb{R}$, the Fourier transforms $\hat{f}(u)=\mathcal{F}[f(x)]$ and $\hat{g}(u)=\mathfrak{F}[g(x)]$ exist. Then,

$$
\int_{-\infty}^{\infty} f^{*}(x) g(x) d x=\frac{1}{2 \pi} \int_{-\infty}^{\infty} \hat{f}^{*}(u) \hat{g}(u) d u .
$$

We need a notation for a reflected strip. If $\nu \in(a, b)$ and $u$ is any real number, then $S$ consists of all $z=u+i \nu$ and $S^{*}$ consists of all $z=u-i \nu$.

THEOREM 3.2. (Option Valuation). Let $V\left(S_{0}\right)$ be the current price of a European-style option with a payoff function $w(x) \geq 0$, where $x=\log S_{T}$. Assume that $w(x)$ is Fourier integrable in $a$ strip and bounded for $|x|<\infty$, with transform $\hat{w}(z), \quad z \in \boldsymbol{S}_{w}$. Let $S_{t}=S_{0} \exp \left[(r-q) t+X_{t}\right]$, where $X_{t}$ is a Lévy process and $\exp \left[X_{t}\right]$ is a martingale. Assume that $X_{T}$ has the analytic characteristic function $\phi_{T}(z)$, regular in the strip $\boldsymbol{S}_{X}=\{z=u+i \nu: \nu \in(a, b)\}$, where $a<-1$ and $b>0$. Then, if $\boldsymbol{S}_{V} \triangleq \boldsymbol{S}_{w} \cap \boldsymbol{S}_{X}^{*}$ is not empty, the option value is given by

$$
V\left(S_{0}\right)=\frac{e^{-r T}}{2 \pi} \int_{i \nu-\infty}^{i \nu+\infty} e^{-i z Y} \phi_{T}(-z) \hat{w}(z) d z, \text { where } \nu=\operatorname{Im} z, \quad z \in \boldsymbol{S}_{V}=\boldsymbol{S}_{w} \cap \boldsymbol{S}_{X}^{*},
$$

and $Y=\ln S_{0}+(r-q) T$. Moreover, $\boldsymbol{S}_{V}$ is not empty when the payoff is a call or put option. 
ProOF: From martingale pricing, since the payoff function is $w\left(\ln S_{T}\right)$, then

$$
\begin{aligned}
V\left(S_{0}\right) & =e^{-r T} \mathbb{E}\left[w\left(\ln S_{T}\right)\right]=\frac{e^{-r T}}{2 \pi} \mathbb{E}\left[\int_{i \nu-\infty}^{i \nu+\infty} S_{T}^{-i z} \hat{w}(z) d z\right], \quad \nu=\operatorname{Im} z, \quad z \in \boldsymbol{S}_{w} \\
& =\frac{e^{-r T}}{2 \pi} \mathbb{E}\left[\int_{i \nu-\infty}^{i \nu+\infty} \exp \left\{-i z\left[\ln S_{0}+(r-q) T\right]\right\} \exp \left(-i z X_{T}\right) \hat{w}(z) d z\right] .
\end{aligned}
$$

In the first line we just inserted the transform representation for $w\left(\ln S_{T}\right)$. In the second line, we inserted $S_{T}=S_{0} \exp \left[(r-q) T+X_{T}\right]$. Next, we bring the expectation inside the integral, which requires an exchange of integration order. This exchange is just made formally here; we validate it by Theorem (3.4) below, an alternative proof of (3.5). Certainly a necessary condition for the exchange to be valid is that $\mathbb{E}\left[\exp \left(-i z X_{\tau}\right)\right]=\phi_{T}(-z)$ exists. Now $\phi_{T}(-z)$ exists if $z \in S_{X}^{*}$, but $z$ is already restricted to $z \in S_{w}$. So the whole integrand exists and is regular if $z \in \boldsymbol{S}_{V}=\boldsymbol{S}_{w} \cap \boldsymbol{S}_{X}^{*}$. Substituting $Y=\ln S_{0}+(r-q) T, \quad$ we have (3.5). Now $\boldsymbol{S}_{X}^{*}=\{u+i \nu: \nu \in(\alpha, \beta)\}$, where $\alpha<0$ and $\beta>1$. So $\boldsymbol{S}_{X}^{*}$ intersects both $\operatorname{Im} z<0$ and $\operatorname{Im} z>1$, which shows that $\boldsymbol{S}_{V}$ is not empty for puts or calls

Next, an alternative proof will show that (3.5) is a consequence of Theorem 3.1. To accomplish that, first let $s_{0}=\ln S_{0}$ and $s_{T}=\ln S_{T}$ and consider the distribution function for the log-stock price to reach a terminal value after the elapse of $T$. Define both the distribution function and its density with

$$
Q_{T}\left(x, s_{0}\right)=\operatorname{Pr}\left\{s_{T}<x \mid s_{0}\right\}=\int_{-\infty}^{x} q_{T}\left(\xi, s_{0}\right) d \xi .
$$

LEMMA 3.3. Equation (3.5) is true if the following two integrals are equal:

$$
\int_{-\infty}^{\infty} q_{T}\left(x_{T}, s_{0}\right) w\left(x_{T}\right) d x_{T}=\frac{1}{2 \pi} \int_{-\infty}^{\infty} \hat{q}_{T}\left(-u-i \nu, s_{0}\right) \hat{w}(u+i \nu) d u, \quad u+i \nu \in \boldsymbol{S}_{w} \cap \boldsymbol{S}_{X}^{*} .
$$

PROOF: Recall from the definition that $\phi_{T}(z)=\int_{\mathbb{R}} \exp (i z x) p_{T}(x) d x$, where $p_{T}(x)$ is the transition density for the Lévy process. Introduce a distribution function for that:

$$
P_{T}(x)=\operatorname{Pr}\left\{X_{T}<x\right\}=\int_{-\infty}^{x} p_{T}(\xi) d \xi .
$$

Now $\quad \operatorname{Pr}\left\{s_{T}<x \mid s_{0}\right\}=\operatorname{Pr}\left\{s_{0}+(r-q) T+X_{T}<x \mid s_{0}\right\}=\operatorname{Pr}\left\{X_{T}<x-Y\right\}$,

recalling that $Y=\ln S_{0}+(r-q) T$. In other words, $Q_{T}\left(x, s_{0}\right)=P_{T}(x-Y)$ and so by differentiating w.r.t. $x$, we have $q_{T}\left(x, s_{0}\right)=p_{T}(x-Y)$. By taking the Fourier transform of both sides of this last identity, we have $\hat{q}_{T}\left(z, s_{0}\right)=\hat{p}_{T}(z) \exp (i z Y)$ for $z$ in some strip, call it $\boldsymbol{S}_{q}$. But, 
of course, $\hat{p}_{T}(z) \equiv \phi_{T}(z)$, so $\phi_{T}(z) \exp (i z Y)=\hat{q}_{T}\left(z, s_{0}\right)$ and the strip is $\boldsymbol{S}_{q}=\boldsymbol{S}_{X}$. Taking $z \rightarrow-z$, we have $\phi_{T}(-z) \exp (-i z Y)=\hat{q}_{T}\left(-z, s_{0}\right)$, where $z \in S_{X}^{*}$. Now the first expression on the right-hand-side of (3.6) may also be written as the (discounted) integral of the transition density and the payoff function. This shows that (3.5) is equivalent to the statement:

$$
V\left(S_{0}\right)=e^{-r T} \int_{-\infty}^{\infty} q_{T}\left(x_{T}, s_{0}\right) w\left(x_{T}\right) d x_{T}=\frac{e^{-r T}}{2 \pi} \int_{i \nu-\infty}^{i \nu+\infty} \hat{q}_{T}\left(-z, s_{0}\right) \hat{w}(z) d z, \quad z \in \boldsymbol{S}_{w} \cap \boldsymbol{S}_{X}^{*} .
$$

Letting $z=u+i \nu$ in this last equation shows that (3.5) is true if (3.7) is true.

THEOREM 3.4 Under the assumptions of Theorem 3.2, equation (3.7) is true; hence, so is (3.5)

Proof: Let $u$ be real. Also, let $\nu_{0}$ be any fixed real number, such that $u+i \nu_{0} \in \boldsymbol{S}_{w} \cap \boldsymbol{S}_{X}^{*}$. Also define $f(x)=q_{T}\left(x, s_{0}\right) \exp \left(\nu_{0} x\right)$ and $g(x)=w(x) \exp \left(-\nu_{0} x\right)$, where $s_{0}$ is fixed and need not be displayed. Note that if $z=u+i v_{0}$, then $\hat{q}_{T}\left(-z, s_{0}\right)=\int \exp \left(-i u x+v_{0} x\right) q_{T}\left(x, s_{0}\right) d x=\hat{f}^{*}(u)$ and $\hat{w}(z)=\int \exp \left(i u x-v_{0} x\right) w(x) d x=\hat{g}(u)$. Thus, proving (3.7) valid is equivalent to proving that, regardless of the choice for $\nu_{0}$ :

$$
\int_{-\infty}^{\infty} f(x) g(x) d x=\frac{1}{2 \pi} \int_{-\infty}^{\infty} \hat{f}^{*}(u) \hat{g}(u) d u, \quad u+i \nu_{0} \in \boldsymbol{S}_{w} \cap \boldsymbol{S}_{X}^{*}
$$

We showed above that $q_{T}\left(x, s_{0}\right)$ is Fourier integrable in $\boldsymbol{S}_{X}$. That is, $\int_{-\infty}^{\infty} q_{T}\left(x, s_{0}\right) \exp (i z x) d x$ $=\int_{-\infty}^{\infty} q_{T}\left(x, s_{0}\right) \exp (i u x-\nu x) d x$ exists for all $z=u+i \nu \in \boldsymbol{S}_{X}$. In particular, choose $u=0$ and $\nu=-\nu_{0}$. Obviously any real part is a valid choice. And $\nu=-\nu_{0}$ is a valid choice because if $\nu_{0}$ is the imaginary part of some number in $S_{X}^{*}$ (which it is), then $-\nu_{0}$ is the imaginary part of some number in $\boldsymbol{S}_{X}$. With that choice, we have shown that $\int_{-\infty}^{\infty} q_{T}\left(x, s_{0}\right) \exp \left(\nu_{0} x\right) d x$ $=\int_{-\infty}^{\infty} f(x) d x$ exists. Since $f(x)$ is a non-negative real, then $\int f(x) d x=\int|f(x)| d x<\infty$; i.e., $f(x) \in L^{1}$. Similarly, both $w(x)$ and $\exp (-\nu x)$ are real and non-negative. Since $w(x)$ is Fourier integrable in the strip $z=u+i \nu \in \boldsymbol{S}_{w}$, then $\int_{-\infty}^{\infty} w(x) \exp (i u x-\nu x) d x<\infty$. Taking $u=0$ and $\nu=\nu_{0}$ is a valid choice here (note that $\nu_{0}$ is also the imaginary part of some number in $\boldsymbol{S}_{w}$ ). So, we have shown that $g(x) \in L^{1}$. In addition, under the assumptions of Theorem 3.2,w(x) is bounded for $|x|<\infty$. Hence $g(x)$ is bounded. We have shown that the requirements of Theorem (3.1) are met and so (3.8), (3.7), and (3.5) are all proven.

We note again that Raible (2000) obtained an integral very similar to (3.5), but he presents it as a mixture of Fourier and two-sided Laplace transforms. Because we use the generalized Fourier transform consistently, our strip condition seems to us much more transparent. 
Contour variations. The general formula (3.5), with $\boldsymbol{S}_{w}$ taken from Table 3.1, provides a starting point formula that has many variations. The variations are obtained by the use of the residue calculus. We stress that we maintain the assumptions of the theorem, which means that $S_{X}^{*}=\{z: \alpha \leq \operatorname{Im} z \leq \beta\}$, where $\alpha<0$ and $\beta>1$. We illustrate with the call option. Then, using the Table 3.1 entries, we see that call option price $C(S, K, T)$ is given by

$$
\begin{gathered}
C(S, K, T)=-\frac{K e^{-r T}}{2 \pi} \int_{i \nu_{1}-\infty}^{i \nu_{1}+\infty} e^{-i z k} \phi_{T}(-z) \frac{d z}{z^{2}-i z}, \quad \nu_{1} \in(1, \beta) \\
\text { using } k=\log \left(\frac{S}{K}\right)+(r-q) T .
\end{gathered}
$$

Since $\beta>1$, the open interval $(1, \beta)$ is not empty and the integral exists. The phase factor uses the dimensionless 'moneyness' $k$, which is also a natural moneyness measure for the implied volatility smile. With that variable, 'at-the-money' means that the forward stock price (the price for delivery at expiration) is equal to the strike.

The integrand in (3.9) is regular throughout $S_{X}^{*}$, except for simple poles at $z=0$ and $z=i$. The pole at $z=0$ has a residue $-K e^{-r T} i /(2 \pi)$ and the pole at $z=i$ has a residue $S e^{-q T} i /(2 \pi)$. Let's move the integration contour to $\nu_{2} \in(0,1)$; then by the residue theorem, the call option value must also equal the integral along $\operatorname{Im} z=\nu_{2}$ minus $2 \pi i$ times the residue at $z=i$. That gives us a first alternative formula

$$
C(S, K, T)=S e^{-q T}-\frac{K e^{-r T}}{2 \pi} \int_{i \nu_{2}-\infty}^{i \nu_{2}+\infty} e^{-i z k} \phi_{T}(-z) \frac{d z}{z^{2}-i z}, \quad \nu_{2} \in(0,1)
$$

This is actually our preferred integration formula for the call option ${ }^{21}$. For example, with $\nu_{2}=1 / 2$, which is symmetrically located between the two poles, this last formula becomes

$$
C(S, K, T)=S e^{-q T}-\frac{1}{\pi} \sqrt{S K} e^{-(r+q) T / 2} \int_{0}^{\infty} \operatorname{Re}\left[e^{i u k} \phi_{T}\left(u-\frac{i}{2}\right)\right] \frac{d u}{u^{2}+\frac{1}{4}} .
$$

Next, we move the contour from (3.9) to $\nu_{3} \in(\alpha, 0)$. Then you pick up both poles. Moreover, the integral along $\operatorname{Im} z=\nu_{3}$ is also the put option formula $P(S, K, T)$, since $\operatorname{Im} z<0$ (see Table 3.1). The net result is simply the put/call parity relation, $C=P+S e^{-q T}-K e^{-r T}$, which is our second alternative formula.

Finally, we can move the contours to exactly $\operatorname{Im} z=1$ and $\operatorname{Im} z=0$. Then the integrals become principal value ${ }^{22}$ integrals and you pick up one-half of the residues. For example, start with the integral in (3.9) written in the form 


$$
-\frac{K e^{-r T}}{2 \pi} \int_{i \nu_{1}-\infty}^{i \nu_{1}+\infty} e^{-i z k} \phi_{T}(-z)\left(\frac{i}{z}-\frac{i}{z-i}\right) d z=I_{1}+I_{2}
$$

Now move $I_{1}$ to exactly $\operatorname{Im} z=0$; the residue theorem combined with letting $z=-u$ yields

$$
I_{1}=\frac{1}{2}(2 \pi i) \frac{K e^{-r T} i}{2 \pi}+\frac{K e^{-r T}}{2 \pi} \mathcal{P} \int_{-\infty}^{\infty} e^{i u k} \phi_{T}(u)\left(\frac{i}{u}\right) d u,
$$

where $\mathcal{P}$ denotes the Cauchy principle value and we have taken $u=-z$. Similarly, move $I_{2}$ to exactly $\operatorname{Im} z=1$; the residue theorem yields

$$
I_{2}=-\frac{1}{2}(2 \pi i) \frac{S e^{-q T} i}{2 \pi}+\frac{K e^{-r T}}{2 \pi} \mathcal{P} \int_{i-\infty}^{i+\infty} e^{-i z k} \phi_{T}(-z)\left(\frac{i}{z-i}\right) d z .
$$

Now let $z=-u+i$, and we have

$$
I_{2}=-\frac{1}{2}(2 \pi i) \frac{S e^{-q T} i}{2 \pi}-\frac{S e^{-q T}}{2 \pi} \mathcal{P} \int_{-\infty}^{\infty} e^{i u k} \phi_{T}(u-i)\left(\frac{i}{u}\right) d u .
$$

The integrals are real and may be simplified by taking the real part. Putting the results together yields the call option price in the 'Black-Scholes' form:

$$
C(S, K, T)=S e^{-q T} \Pi_{1}-K e^{-r T} \Pi_{2},
$$

where $\quad \Pi_{1}=\frac{1}{2}+\frac{1}{\pi} \int_{0}^{\infty} \operatorname{Re}\left[\frac{e^{i u k} \phi_{T}(u-i)}{i u}\right] d u, \quad \Pi_{2}=\frac{1}{2}+\frac{1}{\pi} \int_{0}^{\infty} \operatorname{Re}\left[\frac{e^{i u k} \phi_{T}(u)}{i u}\right] d u$.

The first term $e^{-q T} \Pi_{1}=\partial C / \partial S$ is also the 'delta'. The second term $\Pi_{2}=\operatorname{Pr}\left(S_{T}>K\right)$. These integrands are integrable as $u \rightarrow 0$ because $\phi(u)$ is an analytic characteristic function in a neighborhood of $u=0$ and $u=-i$. For example, near $u=0, \phi(u) \approx 1+u \phi^{\prime}(0)$, where $\left|\phi^{\prime}(0)\right|<\infty$ because of analyticity ${ }^{23}$. Hence, the integrand in $\Pi_{2}$ tends to $\operatorname{Re}\left(-i / u-i \phi^{\prime}(0)+k\right)=\operatorname{Im} \phi^{\prime}(0)+k$ as $u \downarrow 0$, which is finite. Because of the martingale identity $\phi(-i)=1$, the $\Pi_{1}$ integrand is also finite as $u \downarrow 0$. For numerical work (3.11) is more efficient: in the Black-Scholes form (3.12) not only are there two integrations, but the integrand falls off more slowly then (3.11) by a factor of $u$.

As we have shown, the residue calculus provides a general mechanism for obtaining a number of variation on the basic formula, most of which have been obtained before. For example, (3.9) was derived by Carr and Madan (1999) in a specialized attack on the call option. The Black-Scholes form (3.12) was obtained in a more general setting by Bakshi and Madan (2000). A formula of the same style as (3.10) was obtained by Lewis (2000) in a stochastic volatility setting. 


\section{CONCLUSIONS}

The generalization of the Black-Scholes' theory to the martingale pricing theory began a competition in mathematical finance between the PDE approach and the probabilistic approach to solving certain problems. For the simple ones, it's a tie because both methods usually work in a few steps. But for some complicated problems, one method seems to win out ${ }^{24}$. For option valuation under general Lévy processes, the probabilistic approach, in my opinion, is the clear victor. One can introduce PDEs, but to get to the same results is a long trek around - I know this because, in fact, I originally obtained a version of (3.5) from a PDE. 
Table 2.1

Characteristic Functions for Lévy Processes in Stock Price Models

\begin{tabular}{|c|c|c|}
\hline Lévy Process & Characteristic Function: $\phi_{T}(\mathrm{z})=\mathbb{E}\left[\exp \left(i \mathrm{z} X_{T}\right)\right]$ & $\begin{array}{c}\text { Strip of } \\
\text { Regularity } S_{X}\end{array}$ \\
\hline $\begin{array}{l}\text { Lognormal Jump- } \\
\text { diffusion }\end{array}$ & $\exp \left\{i z \omega T-\frac{1}{2} z^{2} \sigma^{2} T+\lambda T\left(e^{i z \alpha-z^{2} \delta^{2} / 2}-1\right)\right\}$ & Entire $z$-plane \\
\hline $\begin{array}{l}\text { Double } \\
\text { Exponential } \\
\text { Jump-diffusion }\end{array}$ & $\exp \left\{i z \omega T-\frac{1}{2} z^{2} \sigma^{2} T+\lambda T\left(\frac{1-\eta^{2}}{1+z^{2} \eta^{2}} e^{i z \kappa}-1\right)\right\}$ & $\begin{aligned}-\frac{1}{\eta}<\operatorname{Im} z<\frac{1}{\eta} \\
0<\eta<1\end{aligned}$ \\
\hline Variance Gamma & $\exp (i z \omega T)\left(1-i z \nu \theta+\frac{1}{2} \sigma^{2} \nu z^{2}\right)^{-T / \nu}$ & $\begin{array}{l}\beta-\alpha<\operatorname{Im} z<\beta+a \\
\quad \text { (see notes) }\end{array}$ \\
\hline $\begin{array}{l}\text { Normal Inverse } \\
\text { Gaussian }\end{array}$ & $\exp \left\{i z \omega T+\delta T\left[\sqrt{\alpha^{2}-\beta^{2}}-\sqrt{\alpha^{2}-(\beta+i z)^{2}}\right]\right\}$ & $\beta-\alpha<\operatorname{Im} z<\beta+a$ \\
\hline $\begin{array}{l}\text { Generalized } \\
\text { Hyperbolic }\end{array}$ & $\exp (i z \omega T)\left(\frac{\left(\alpha^{2}-\beta^{2}\right)}{\alpha^{2}-(\beta+i z)^{2}}\right)^{\lambda T / 2}\left(\frac{K_{\lambda}\left(\delta \sqrt{\alpha^{2}-(\beta+i z)^{2}}\right.}{K_{\lambda}\left(\delta \sqrt{\alpha^{2}-\beta^{2}}\right)}\right.$ & $\beta-\alpha<\operatorname{Im} z<\beta+a$ \\
\hline $\begin{array}{l}\text { Finite Moment } \\
\text { Logstable }\end{array}$ & $\exp \left\{i z \omega T-(i z \sigma)^{\alpha} T \sec \frac{\pi \alpha}{2}\right\}$ & $\operatorname{Im} z<0$ \\
\hline
\end{tabular}

Notes: The stock price evolution is $S_{T}=S_{0} \exp \left[(r-q) T+X_{T}\right]$, where $r$ is the interest rate, $q$ is the dividend yield, and $X_{T}$ is a Lévy process. For the Variance Gamma model, the strip of regularity uses $\alpha=\left[2 /\left(\nu \sigma^{2}\right)+\left(\theta^{2} / \sigma^{4}\right)\right]^{1 / 2}, \beta=\theta / \sigma^{2}$. For each model, the drift parameter $\omega$ is determined by $\phi_{T}(-i)=1$. For the Variance Gamma, Normal Inverse Gaussian, and Generalized Hyperbolic models, we need $\alpha+\beta \geq 0$ and $\beta-\alpha \leq-1$ for a good stock market model. 
Table 3.1

Generalized Fourier Transforms for Various Financial Claims

\begin{tabular}{|c|c|c|c|}
\hline $\begin{array}{l}\text { Financial } \\
\text { Claim } \\
\text { (Option) }\end{array}$ & $\begin{array}{c}\text { Payoff } \\
\text { Function: } \\
w(x)\end{array}$ & $\begin{array}{c}\text { Payoff } \\
\text { Transform: } \\
\hat{w}(z)=\mathcal{F}[w(x)]\end{array}$ & $\begin{array}{c}\text { Strip } \\
\text { of } \\
\text { regularity } S_{w}\end{array}$ \\
\hline Call option & $\left(e^{x}-K\right)^{+}$ & $-\frac{K^{i z+1}}{z^{2}-i z}$ & $\operatorname{Im} z>1$ \\
\hline Put option & $\left(K-e^{x}\right)^{+}$ & $-\frac{K^{i z+1}}{z^{2}-i z}$ & $\operatorname{Im} z<0$ \\
\hline $\begin{array}{l}\text { Covered call or } \\
\text { cash-secured put }\end{array}$ & $\min \left(e^{x}, K\right)$ & $\frac{K^{i z+1}}{z^{2}-i z}$ & $0<\operatorname{Im} z<1$ \\
\hline Delta function & $\delta(x-\ln K)$ & $K^{i z}$ & Entire $z$-plane \\
\hline Money market & 1 & $2 \pi \delta(z)$ & $\operatorname{Im} z=0$ \\
\hline
\end{tabular}

Notes: The table uses $x=\log S_{T}$, where $S_{T}$ is the security price at expiration, and $x^{+}=\max (x, 0)$. The parameter $K$ is a strike (exercise) price. The expression $\delta\left(x-x_{0}\right)$ is the Dirac delta function. The symbol $\mathfrak{F}[\cdot]$ indicates taking a Fourier transform. 


\section{References}

Aase, Knut K.(1988): Contingent Claims Valuation when the Security Price is a Combination of

an Ito Process and a Random Point Process, Stochastic Processes and their Applications, 28, $185-220$.

Bakshi, Gurdip and Dilip Madan (2000): Spanning and Derivative-security Valuation, J. of Financial Economics, 55, 205-238.

Barndorff-Nielsen, O.E., T Mikosch, and S. I. Resnick, eds (2001): Levy Processes: Theory and Applications, Birkhäuser, Boston.

Barndorff-Nielsen, O.E (1998): Processes of Normal Inverse Gaussian Type, Finance and Stochastics, 2, 41-68.

Bates, David S. (1988): Pricing options on jump-diffusion processes. Working paper 37-88, Univ. of Pennsylvania, Rodney L. White Center.

Bates, David S. (1991): The Crash of '87: Was it Expected? The Evidence from Options Markets, J. Finance 46, July, 1009-1044.

Bertoin, Jean (1996): Lévy Processes, Cambridge University Press.

Bibby, Bo Martin and Michael Sørensen (2001): Hyperbolic Processes in Finance, manuscript, Institute of Mathematical Sciences, Univ. of Copenhagen.

Breiman, Leo (1992), Probability, SIAM, Philadelphia.

Bremaud, Pierre (1974): The Martingale Theory of Point Processes over the Real Half Line Admitting an Intensity. Control Theory, Numerical Methods and Computer System Modelling. Series: Lecture Notes in Economics and Mathematical Systems, Vol. 107, Springer-Verlag, Berlin, 519-542.

Carr, Peter and Dilip B. Madan (1999): Option Valuation using the Fast Fourier Transform, J. Computational Finance, 2, No.4, Summer, 61-73. 
Carr, Peter and Liuren Wu (2000): The Finite Moment Logstable Process and Option Pricing, manuscript, Feb. 21, 2000.

Colwell, David B. and Robert J. Elliott (1993): Discontinuous Asset Price and Non-Attainable Contingent Claims, Math. Finance, 3, (July), 295-308.

Eberlein, Ernst, Ulrich Keller, and Karsten Prause (1998): New Insights into Smile, Mispricing, and Value at Risk: the Hyperbolic Model, J. Business, 71, 371-405.

Kou, S.G. (2000): A Jump Diffusion Model for Option Pricing with Three Properties: Leptokurtic Feature, Volatility Smile, and Analytical Tractability, preprint, Columbia University, Feb. 2000.

Lewis, Alan L. (1998): Applications of Eigenfunction Expansions in Continuous-Time Finance, Math. Finance, 8, No.4, October 1998, 349-383

Lewis, Alan L. (2000): Option Valuation under Stochastic Volatility, Finance Press, Newport Beach, California.

Lillestøl, Jostein (1998). Fat and Skew: Can NIG Cure? On the Prospects of Using the Normal Inverse Gaussian Distribution in Finance, June 15, 1998 manuscript, The Norwegian School of Economics and Business Administration.

Madan, Dilip B., Peter Carr, and Eric C. Chang (1998): The Variance Gamma Process and Option Pricing, manuscript, University of Maryland, June 1998 (forthcoming, European Finance Review).

Merton, R.C.(1976): Option Pricing When Underlying Stock Returns are Discontinuous, J. of Financial Economics, 3, Jan-Mar, 125-144. Reprinted as Ch. 9 in Continuous-Time Finance. Basil Blackwell, Cambridge Mass (1990).

Naik, Vasanttilak and Moon Lee (1990): General Equilibrium Pricing of Options on the Market Portfolio with Discontinuous Returns, The Review of Financial Studies, 3, number 4, 493-521.

Prause, Karsten (1999): The Generalized Hyperbolic Model: Estimation, Financial Derivatives, and Risk Measures, Dissertation, Faculty of Mathematics, Univ. of Freiburg, Germany 
Raible, Sebastian (2000): Lévy Processes in Finance: Theory, Numerics, and Empirical Facts, Dissertation, Faculty of Mathematics, Univ. of Freiburg, Germany.

Sato, Ken-Iti (1999): Lévy Processes and Infinitely Divisible Distributions. Cambridge University Press.

Titchmarsh, E.C. (1975): Introduction to the Theory of Fourier Integrals, Oxford University Press. Reprint of the 1948 second edition. 


\section{Notes}

${ }^{1}$ First, when there is the possibility of a significantly negative jump (a 'crash'), then expiring out-of-the-money puts become worthless like $\lambda T$. (see Merton's 1976 series solution). Here $\lambda$ is the jump arrival rate under the martingale pricing distribution, and $T$ is the time to option expiration. This is a much slower decay in $T$ than the Black-Scholes formula, and if you try to fit the Black-Scholes formula to it (with an implied volatility), the only way it works is for the implied volatility to grow very large as $T \rightarrow 0$. Second, the jump rate $\lambda$ is very sensitive to preferences. Statistically, you might expect a market crash once every 10 years for example $(\lambda=0.10)$. But since you are risk-averse, your effective rate for $\lambda$, which you use to price options is once every 4 years for another example $(\lambda=0.25)$. These two effects both work in the same direction to substantially boost the implied volatility above the statistical volatility of the index. Very steep smiles are easy to fit because the model generates arbitrarily large implied volatilities as $T \rightarrow 0$. For preferences effects in Merton's model, see Bates (1991,1998) and Naik and Lee (1990). For general measure changes in jump-diffusions, see Aase (1998) and Colwell and Elliott (1993).

${ }^{2}$ The independent increment property is too idealized for the real world. For example, while actual security returns have very low autocorrelations, squared or absolute returns often have significant positive autocorrelations, and volatility clustering effects are well-documented. These effects are missing from a pure Lévy process model.

${ }^{3}$ See, for example, the $S$-space call option formula obtained by Madan, Carr, and Chang (1998).

${ }^{4}$ Proportional returns problems, which may have many stochastic factors, have $d S_{t} / S_{t}$ independent of $S_{t}$.

${ }^{5}$ Equivalently, this transform is a complex Mellin transform using $S_{T}$ as the integration variable.

${ }^{6}$ We tested our results in the Mathematica system, where (1.1) is generally set up in just a few lines of code and takes typically a couple seconds (on an old desktop system) to evaluate any of the half-dozen Lévy examples from the literature. Since Mathematica is an interpretive system, this is a worst case scenario for run-times. When the highest numerical efficiency is important, consider applying the Fast Fourier Transform method of Carr and Madan (1999) to (1.1) or any of the variants given in Sec. 3.

${ }^{7}$ For example, the change of measure induced by an equilibrium model with a power utility function $u^{\prime}(S)=S^{-\theta}$ is very popular, often applied in an ad-hoc manner as an Esscher transform. This particular measure change causes a simple translation $z \rightarrow z+i \theta$ in the Fourier transform of the Lévy measure $\hat{\mu}(z)$, defined below. Some, but not all of the models in Table 2.1 can maintain their parameterized form under this particular change of measure.

${ }^{8}$ A cumulative probability distribution $F(x)$ is any non-decreasing function with $F(-\infty)=0$ and $F(\infty)=1$.

${ }^{9}$ This is a rearrangement of terms in Jacod and Shiryaev (1987) Theorem II.2.34. 
${ }^{10}$ Sometimes the term complex Fourier transform is used. A comprehensive reference is Titchmarsh (1975). The reason for the limitation to the strip $a<\operatorname{Im} z<b$ will be explained below.

11 Write $X_{t}=\sum\left(X_{i \Delta t}-X_{(i-1) \Delta t}\right)$, where $t=n \Delta t$. For simplicity, take $z$ to be real. We can compute $\phi_{t}(u)=\mathbb{E}\left[\exp \left(i u X_{t}\right)\right], u \in \mathbb{R}$, as a product of $n$ independent expectations because $X_{t}$ has independent increments. Each individual term is identical: $\int_{\mathbb{R}} \exp (i u x) p_{\Delta t}(x) d x$, and exists because $u$ is real and $p$ is a probability density.

${ }^{12}$ See, for example, Breiman (1992), p.310 or Bremaud (1974).

${ }^{13}$ This is Bertoin's form; some authors call $\Psi(-i u)$ or $-\Psi(-i u)$ the characteristic exponent. The general expression follows simply from the same argument in footnote 11 .

${ }^{14}$ Warning: there is no uniformity in the finance literature regarding the normalization of $\phi(z)$ at $z=-i$.

${ }^{15}$ A function $f(z)$ is analytic at a complex-valued point $z$ if it has a derivative there. If it's both analytic and singlevalued in a region, it's called regular.

${ }^{16}$ This is not always the case in other proportional returns models such as stochastic volatility models, where the strip of regularity of the characteristic function of $\log S_{t}$ can depend upon $t$. [see Lewis, 2000, p.55]

17 Any SDE of the form $d S_{t}=S_{t^{-}} d Z_{t}$, where $Z_{t}$ is a given semimartingale can be integrated by writing $Z_{t}=Z_{t}^{c}+Z_{t}^{d}$, a continuous part and a purely discontinuous part. Then, apply the Doléans-Dade exponential formula (see Jacod and Shiryaev, 1987, Theorem 4.61):

$$
S_{t}=\exp \left(Z_{t}-\frac{1}{2}\left\langle Z^{c}, Z^{c}\right\rangle_{t}\right) \prod_{0<s \leq t}\left(1+\Delta Z_{s}\right) e^{-\Delta Z_{s}} .
$$

${ }^{18}$ The characteristic function for Merton's model was previously obtained by Carr and Wu (2000).

${ }^{19}$ Of course, in principle, the representation can always be used, but sometimes the Lévy measures are very complicated and the formula is not helpful

${ }^{20}$ By Cauchy's Theorem, the integration contour can be deformed as long as it remains within the strip of regularity and extends to $\pm \infty$.

${ }^{21}$ I checked the formula numerically and found agreement with several known results: (i) the power series in $\lambda$ solution of Merton's lognormal jump-diffusion; (ii) the special function solution for the double exponential model, using the Mathematica code that S.G. Kou has made available on the Internet; (iii) The finite moment logstable model, using some high precision call option results graciously supplied by Liuren Wu and Peter Carr.

${ }^{22}$ The Cauchy principal value is defined by 


$$
\mathcal{P} \int_{a}^{b} \frac{f(u)}{u-u_{0}} d u=\lim _{\varepsilon \rightarrow 0}\left[\int_{a}^{u_{0}-\varepsilon} \frac{f(u)}{u-u_{0}} d u+\int_{u_{0}+\varepsilon}^{b} \frac{f(u)}{u-u_{0}} d u\right] \text {, where } \varepsilon \text { is positive }
$$

${ }^{23}$ Analyticity will fail, although the final result may still be valid in borderline cases where $S_{X}^{*}=\{z: a \leq \operatorname{Im} z \leq 1\}$ or $S_{X}^{*}=\{z: 0 \leq \operatorname{Im} z \leq b\}$. For example, the finite moment logstable model is not analytic at $z=0$, but $\left|\phi^{\prime}(0)\right|<\infty$ for $1<\alpha<2$ (see Table 2.1). Hence (3.12) exists in that case, but we make no general claims and leave those cases as an open question

${ }^{24}$ For example, a pure probabilistic approach might have difficulty solving the two problems in Lewis (1998). 